\title{
ASSOCIATED ALGEBRAIC AND PARTIAL DIFFERENTIAL EQUATIONS
}

BY J. A. GREENWOOD

1. Introduction. Between an algebraic equation and an ordinary linear homogeneous differential equation with constant coefficients, in a single unknown, there is a familiar and useful association, illustrated by the equations

$$
x^{2}+3 x-4=0, \quad u^{\prime \prime}+3 u^{\prime}-4 u=0 .
$$

Riquier* has shown that a similar association exists between finite systems consisting of $m$ algebraic equations in $n$ unknowns and $m$ linear homogeneous partial differential equations in a single unknown and $n$ independent variables as follows. Let

$$
\sum_{0}^{p} a_{i_{1} \cdots i_{n}}^{\alpha}\left(i_{1} \cdots i_{n}\right)=0, \quad(\alpha=1, \cdots, m),
$$

be any system of algebraic equations, where the $a$ 's are constants and $\left(i_{1} \cdots i_{n}\right)$ is to be interpreted as the monomial $x_{1}{ }^{i_{1}} \cdots x_{n}{ }^{i_{n}}$. Let the associated system of partial differential equations be

$$
\sum_{0}^{p} a_{i_{1} \cdots i_{n}}^{\alpha}\left(i_{1} \cdots i_{n}\right) u=0, \quad(\alpha=1, \cdots, m),
$$

where $\left(i_{1} \cdots i_{n}\right)$ is to be interpreted as the differential operator $\partial^{i_{1}+\cdots+i_{n}} / \partial x_{1} i_{1} \cdots \partial x_{n}{ }^{i n}$.

We shall prove in this paper the two following theorems.

THEOREM 1. System (1) is inconsistent if and only if the general solution of (2) is $u=0$.

THEOREM 2. The general solution of (2) is a non-zero polynomial if and only if $x_{1}=\cdots=x_{n}=0$ is the solution of (1).

2. Corresponding Operations on the Two Systems. We shall mul-

* Sur la résolution numérique du système d'équations algébriques entières à un nombre quelconque d'inconnues, Annales Scientifiques de l'École Normale Supérieure, vol. 63 (1928), pp. 145-188. 
tiply the equations by $\left(j_{1} \cdots j_{\nu}\right)$ and then form linear combinations of them with constant coefficients. The interpretation of the $\left(j_{1} \cdots j_{v}\right)$ is to be that of the system into which it is multiplied. This gives rise to new associated systems of equations.

3. System (1) Inconsistent. Let us assume (1) is inconsistent. Then according to a known theorem* there exist for system (1) $C$ 's satisfying the relation

$$
1 \equiv \sum_{\alpha}^{1, \cdots, m} C^{\alpha}\left(\sum_{0}^{p} a_{i_{1} \cdots i_{n}}^{\alpha}\left(i_{1} \cdots i_{n}\right)\right),
$$

where

$$
C^{\alpha}=\sum_{0}^{r} k_{i_{1} \cdots i_{n}}^{\alpha}\left(i_{1} \cdots i_{n}\right) .
$$

The corresponding result on (2) would of course be obtained by multiplying both sides of the equations (3) on the right by $u$. Then (2) implies $u=0$ and from the form of (2) it is seen to be an actual solution.

If (1) has a solution $\left(\bar{x}_{1}, \cdots, \bar{x}_{n}\right)$, then (2) has the obvious immediate $\dagger$ solution $c \exp \left(\bar{x}_{1} x_{1}+\cdots+\bar{x}_{n} x_{n}\right)$, where $c$ is an arbitrary constant. On the assumption that $u=0$ is the general solution of (2), it follows immediately from this that (1) must be inconsistent. Thus Theorem 1 is established.

4. System (1) Consistent with only the Trivial Solution. Now consider the case where $x_{1}=\cdots=x_{n}=0$ is the solution of (1). According to Hilbert's zero theorem there exist $C$ 's similar to (4) and positive integers $\rho_{j}$ such that

$$
\begin{aligned}
x_{j}^{\rho_{j}} \equiv \sum_{\alpha} C^{\alpha_{j}}\left(\sum_{0}^{p} a_{i_{1} \cdots i_{n}}^{\alpha}\left(i_{1} \cdots i_{n}\right)\right), \\
\quad(\alpha=1, \cdots, m ; j=1, \cdots, n) .
\end{aligned}
$$

Again, multiplying both sides of (5) on the right by $u$ and interpreting $x_{j}{ }^{{ }^{j}}$ as in (2), we see that the left member becomes $\partial^{\rho j} u / \partial x_{j}{ }^{\rho_{i}}$. System (2) implies that these derivatives vanish

* B. L. Van der Waerden, Moderne Algebra, vol. 1, 1930, p. 10.

$\dagger$ Riquier, loc. cit. 
identically. Observe that there must be a non-zero solution of (2) else we have a contradiction with Theorem 1.

Consider any derivative $\left(i_{1} \cdots i_{n}\right)$ whose order equals or exceeds $\rho_{1}+\cdots+\rho_{n}$, that is,

$$
\left(i_{1}-\rho_{1}\right)+\cdots+\left(i_{n}-\rho_{n}\right) \geqq 0 .
$$

Then $i_{j} \geqq \rho_{j}$ for at least one $j$. Hence every derivative of the order considered is zero. Since there are but a finite number of derivatives below a given order taken with respect to a finite number of variables, it is clear that the series expansion solution for $u$ reduces to a non-zero polynomial. If we assume that a nonzero polynomial is the general solution of (2), then (1) cannot have more than the trivial solution. For if it did, (2) would have a non-constant exponential solution. But if (1) has no solution our present hypothesis contradicts Theorem 1. Therefore (1) has the trivial solution and that alone, and Theorem 2 is proved.

We give an example illustrating the second result. Let (1) be

$$
x_{1}^{3}+x_{2}{ }^{3}=0, \quad x_{1}^{3}-x_{2}{ }^{3}=0,
$$

and its associated system of partial differential equations (2) be

$$
\frac{\partial^{3} u}{\partial x_{1}{ }^{3}}+\frac{\partial^{3} u}{\partial x_{2}{ }^{3}}=0, \quad \frac{\partial^{3} u}{\partial x_{1}{ }^{3}}-\frac{\partial^{3} u}{\partial x_{2}{ }^{3}}=0 .
$$

Clearly $\rho_{1}=\rho_{2}=3$. Thus the series expansion for $u$ reduces to

$$
u=\sum_{0}^{2} a_{i_{1} i_{2}} x_{1}^{i_{1}} x_{2}^{i_{2}},
$$

where the coefficients are arbitrary constants.

An interesting application of the results obtained in this paper is that the conditions given by Riquier* for the existence of a solution of a system of algebraic equations (1) can now be extended to read: The system (1) is consistent if and only if there exists a non-zero solution of (2). In other words, if (2) has any non-zero solution, it also has a non-zero immediate solution.

Duke UnIVERsity

* Riquier, loc. cit. 